\title{
Optimization of analysis methods for heavy metals in sediment for the trace element geochemistry of the Bay of Bengal Coast, Bangladesh
}

\author{
MST SHAMSUN MIWA NAHAR
}

Eurofins Earth Consultant Co. Ltd (eurofins)

Presenting Author: msnahar@yahoo.com

To investigate of heavy metals in sediments, there is a need for optimization of the procedures by evaluating the soil nature. Emphasis is placed on determination of priority pollutant heavy metals (As, $\mathrm{Cr}, \mathrm{Cd}, \mathrm{Pb}, \mathrm{Cu}, \mathrm{Zn}$, REEs) in sediments from the trace amount to high content. Generally heavy trace metals in sediments are quantified by the conventional process in aqueous media or in solid phase. Two methods include X-ray fluorescence (XRF) and inductively coupled plasma mass spectrometry (ICP-MS) analyses. It is observed the dissimilarities of the result were investigated for the different optical method using XRF for the solid soil and ICP-MS for the digested soil solution. ICP-MS offers the high sensitivity analysis with lower detection limits of most elements are in ppt level and isotopic analysis can be achieved in aqueous media only. However it has been difficult to develop the dissolution techniques for heavy metals in all forms of soil by using temperature, pressure in alkali, or in acid, medium due to sediment structure. For to this reason, some percent of heavy metals remain in residue during sediment digestion process. Moreover, it has been difficult to determine the key trace metals at the same time due to contamination and interference from major constituents in sediment solution. Therefore, it should be clarifying the method for getting the reliable data values for the actual metal content in sediment sample. A new mixture type of reference soil (heavy metals: $1-20000 \mathrm{ppm}$ ) for X-ray diffraction (XRF) method was used for quantitative estimation of selective sediment minerals. A two-step optimized determination procedure, in the sequence metal quantification by mixture type XRF reference soil in solid form and comparing the data with the ICP-MS data obtained from digested sediment and residue, is proposed. The results offer novel insights into the analysis method for heavy metals in soil or sediment samples. This research first investigated the distribution pattern of rare-earth concentration in coastal sediment, providing a baseline in the Bay of Bengal, Bangladesh. We have, therefore, drawn new color maps for As, heavy metals and rare-earth and found the current geochemical pattern. 